\title{
Outstanding Contributions to Logic
}

\author{
Volume 10
}

\section{Editor-in-Chief}

Sven Ove Hansson, Royal Institute of Technology, Stockholm, Sweden

\section{Editorial Board}

Marcus Kracht, Universität Bielefeld

Lawrence Moss, Indiana University

Sonja Smets, Universiteit van Amsterdam

Heinrich Wansing, Ruhr-Universität Bochum 
More information about this series at http://www.springer.com/series/10033 
Eugenio G. Omodeo - Alberto Policriti Editors

Martin Davis

on Computability,

Computational Logic, and

Mathematical Foundations

黛 Springer 


\section{Editors}

Eugenio G. Omodeo

Alberto Policriti

University of Trieste

University of Udine

Trieste

Italy

Udine

Italy

ISSN 2211-2758

ISSN 2211-2766 (electronic)

Outstanding Contributions to Logic

ISBN 978-3-319-41841-4

ISBN 978-3-319-41842-1 (eBook)

DOI 10.1007/978-3-319-41842-1

Library of Congress Control Number: 2016954526

(C) Springer International Publishing Switzerland 2016

This work is subject to copyright. All rights are reserved by the Publisher, whether the whole or part of the material is concerned, specifically the rights of translation, reprinting, reuse of illustrations, recitation, broadcasting, reproduction on microfilms or in any other physical way, and transmission or information storage and retrieval, electronic adaptation, computer software, or by similar or dissimilar methodology now known or hereafter developed.

The use of general descriptive names, registered names, trademarks, service marks, etc. in this publication does not imply, even in the absence of a specific statement, that such names are exempt from the relevant protective laws and regulations and therefore free for general use.

The publisher, the authors and the editors are safe to assume that the advice and information in this book are believed to be true and accurate at the date of publication. Neither the publisher nor the authors or the editors give a warranty, express or implied, with respect to the material contained herein or for any errors or omissions that may have been made.

Printed on acid-free paper

This Springer imprint is published by Springer Nature

The registered company is Springer International Publishing AG

The registered company address is: Gewerbestrasse 11, 6330 Cham, Switzerland 
This book is dedicated to the memory of Hilary Putnam. 


\section{Foreword}

I am honored to write a brief foreword to this volume dedicated to the many logical issues on which Martin has shed light during his illustrious career. I first met Martin at the Cornell 1957 Summer Institute in Symbolic Logic. This meeting proved very significant to the mathematical lives of many people who met there, including both of us: for him, Hilary Putnam and for me, J. Barkley Rosser. This meeting precipitated the formation of an international research community in mathematical logic whose influence on logic and computer science is strong even after sixty years. What I learned then about Martin was the universality of his interests, his utter concentration on fundamental problems, and his insatiable urge to learn new things. These are the signal marks of his long career. Some years ago, a university in a Western state with no history in any area he represents and, without warning, offered him a prestigious post. He called me and asked why. I told him they wanted an icon. He said he did not want to be an icon and promptly turned it down. But he is an icon, whether he likes it or not! 


\section{Preface}

\begin{abstract}
It is reasonable to hope that the relationship between computation and mathematical logic will be as fruitful in the next century as that between analysis and physics in the last. The development of this concern demands a concern for both applications and mathematical elegance.
\end{abstract}

(John McCarthy 1963)

The dozen students or so gathered in the lovely Italian town of Perugia were amazed when Martin David Davis first showed up, wearing heavy shoes and short pants seemingly more apt for trekking than for teaching a graduate level course. His accent from the Bronx, together with a little hole, may be produced by the ember of a cigarette, over one shoulder of his red T-shirt, seized the attention of the class. His hair $\grave{a}$ la Queen of Sheba further increased the mismatch between Martin as a person and the stereotype unavoidably associated with his reputation as a distinguished scholar.

Admiration quickly prevailed over astonishment when Martin began his exposition of computability. For the entire one-month duration of his course, concepts remained clear and accessible. At times, when confronted with some odd question coming from his audience, Martin turned his hands upward and disarmingly said "I cannot understand"; far more often, he answered with extreme precision. He indulged in vivid images, such as "brand-new variable" or "crystal-clear proof," but his repertoire of idiomatic expressions also included "gory detail," when technicalities were inescapable.

Through mathematics, the class felt, Martin was also addressing issues of philosophical relevance and depth. On one memorable occasion, the philosophical side of his scientific inquiry showed through a lesson boldly offered in Italian. Forty years have elapsed since, and alas, the tape recording of that lesson, dealing with Turing machines and universality, has by now faded away.

Once Martin was invited to an assembly of all students participating in the Perugia summer school. Unhesitatingly, he joined the crowd, coming hand in hand 
with his wife Virginia; he even took the floor at some point, rational and quiet, not in the least dismayed by the excited, somehow "revolutionary," atmosphere of the event.

One of the editors of this volume dedicated to Martin was a student in his computability course in Perugia, and this explains why such an anecdotal episode has been recounted here. Many similar fascinating stories could certainly be reported by many: Over the years, Martin lectured in several countries (to cite a few: Japan, India, England, Russia, and Mexico—see Fig. 1), and they have_-along

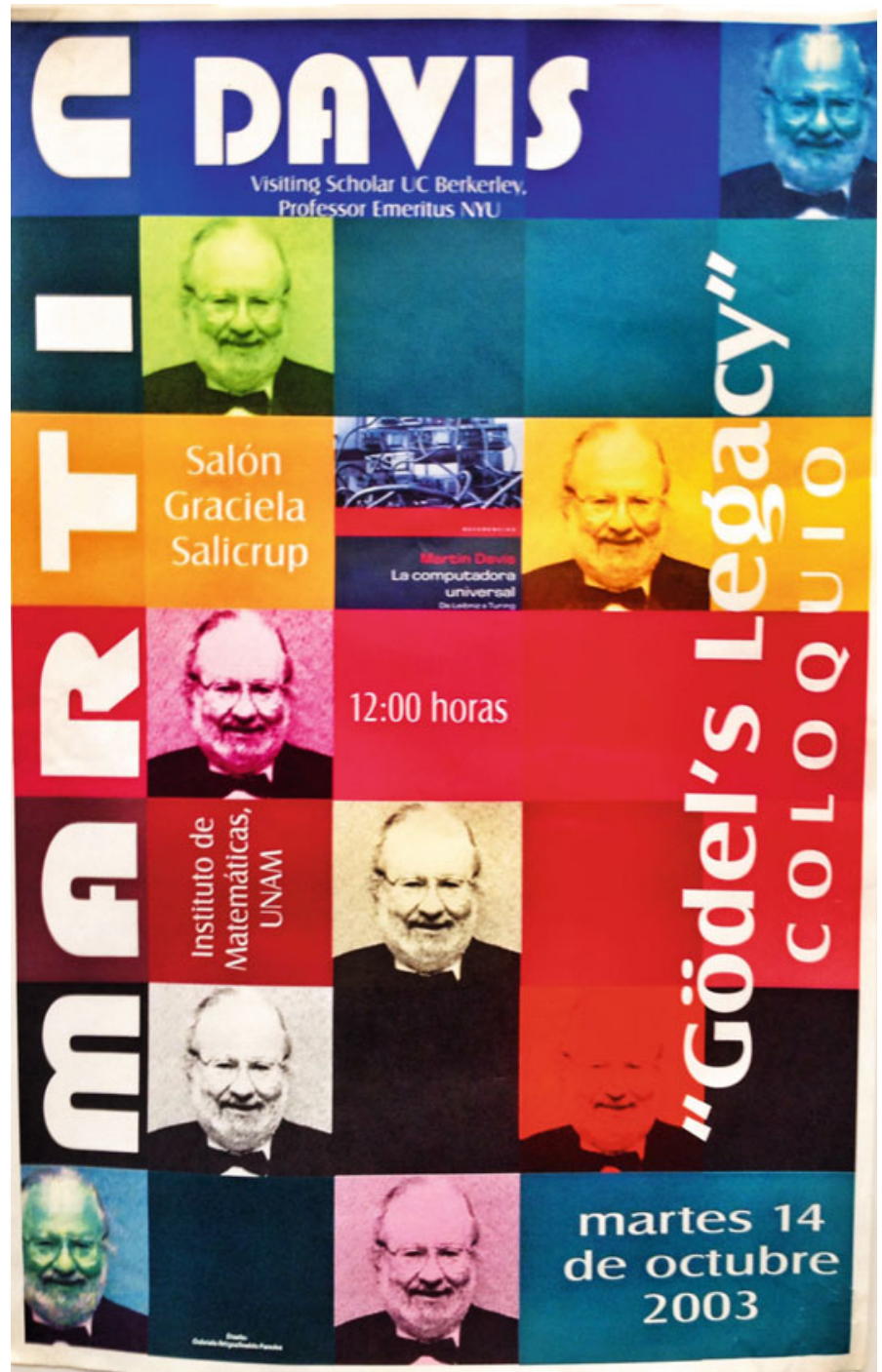

Fig. 1 Poster announcing Martin's first lecture in Mexico 
with his publications - exerted a wide influence. This book will testify to this influence by focusing on scientific achievements in which Martin was involved in the first person and on further achievements, studies, and reflections in which work and vision consonant with his have played a role. Our task has been to collect testimonies of Martin's contributions to computability, computational logic, and mathematical foundations.

Three chapters are devoted to a problem that Martin said he found "irresistibly seductive" when still an undergraduate (Fig. 2) and which progressively became his "lifelong obsession": Hilbert's tenth problem-H10 for short. One of the three contains a narrative essay by the Mexican mathematician Dr. Laura Elena Morales Guerrero, telling us how a negative solution to H10 came to light through the joint effort of four protagonists (one being Martin, of course). There are two epic events in that story: One is when Julia Bowman Robinson eliminates "a serious blemish" from a proof by Martin and Hilary Putnam by showing how to avoid a hypothesis that was unproved at the time; the other is when, in 1970, notes of a talk given in Novosibirsk reach Martin in New York. Laura Elena reports to the reader the key equations in those notes based on Yuri Matiysevich's decisive work, which Martin echoed a few months later by his own use of these newly developed methods to obtain an alternative system of equations leading to the same result. See Fig. 3.

If we could also prove that the olase of diophentine predicates is olosed under bounded universal quantiflcation, then it would follow from 2.7 that every recursively enumerable predicate is diophantine.

But this olearly would lead to the unsolvability of the general decision problem for diophantine equations in non-negative 1ntegers, and hence--by the rerarks of $\$ 1-0$. Hilbert's tenth problen.

Now, evidently every diophantine predicate is rooursively enumerable. If wo also know the converse of this result, then wo should of cours be able to answer the above question in the affirmative, with all the attendent consequences. Hence our investigetion of the reletion between Q1ophentine prediostes and recursitely onumersblo predicates.

Fig. 2 Are all recursively enumerable sets Diophantine? (From Martin's Ph.D. thesis) 
Fig. 3 In all of these equations, variables range over $\mathbb{N}$. Equations (I)-(X) with parameters $u, v, a$ have a solution for $a>1$ if and only if $v=y_{u}(a)$, where $X=$ $x_{u}(a), Y=y_{u}(a)$ is the $u+1$ st solution, over $\mathbb{N}$, of the Pell equation $X^{2}-\left(a^{2}-1\right) Y=1$. Equations (I)-(XV) with parameters $\alpha, \beta, u$ have a solution for $\beta \geq 1$ if and only if $\alpha=\beta^{u}$

\begin{tabular}{|c|c|}
\hline (I) & $u+j=v$ \\
\hline$\left(\mathrm{II}_{\mathrm{a}}\right)$ & $p+(a-1) q=v+r+1$ \\
\hline$\left(\mathrm{II}_{\mathrm{b}}\right)$ & $g=v+t+1$ \\
\hline (III) & $p^{2}-\left(a^{2}-1\right) q^{2}=1$ \\
\hline$\left(\mathrm{IV}_{\mathrm{a}}\right)$ & $h+(a+1) g=b(p+(a+1) q)^{2}$ \\
\hline$\left(\mathrm{IV}_{\mathrm{b}}\right)$ & $h+(a-1) g=c(p+(a-1) q)^{2}$ \\
\hline$(\mathrm{V})$ & $h^{2}-\left(a^{2}-1\right) g^{2}=1$ \\
\hline (VI) & $m=(h+(a+1) g) z+a$ \\
\hline (VII) & $m=(p+(a-1) q) f+1$ \\
\hline (VIII) & $x^{2}-\left(m^{2}-1\right) y^{2}=1$ \\
\hline (IX) & $y=d(p+(a-1) q)+u$ \\
\hline$(\mathrm{X})$ & $y=e(h+(a+1) g)+v$ \\
\hline (XI) & $w^{2}-\left(a^{2}-1\right) v^{2}=1$ \\
\hline (XII) & $(w-(a-\beta) v-\alpha)^{2}=\gamma^{2}\left(2 a \beta-\beta^{2}-1\right)^{2}$ \\
\hline (XIII) & $\alpha+\tau+1=2 a \beta-\beta^{2}-1$ \\
\hline$(\mathrm{XIV})$ & $\eta=\beta+\zeta+1=u+\xi+1$ \\
\hline$(\mathrm{XV})$ & $a^{2}-\left(\eta^{2}-1\right)(\eta-1)^{2}(\delta+1)^{2}=1$ \\
\hline
\end{tabular}

Another chapter on H10 is by Yuri V. Matiyasevich himself, the "clever young Russian" whose appearance Martin had predicted and who, by producing a Diophantine predicate of exponential growth, first obtained that negative solution. Yuri explains how Julia Robinson, Martin Davis, and Hilary Putnam had - through extraordinary insights - paved the way for his decisive mathematical contribution. Far from being exhausted, the field of research triggered by H10 abounds today with unanswered questions, some fairly old (e.g., does the equation of the report shown in Fig. 4 admit finitely many solutions?), other quite contemporary, and in fact, Yuri's article, after disclosing a formidable landscape of open issues in front of us, terminates with a conjecture raised by Martin in 2010 .

A third related contribution is by Prof. Alexandra Shlapentokh, who enriches the landscape with extensions of $\mathrm{H} 10$ to recursive rings. When referring his Tenth Problem to integers, in 1900, Hilbert may have thought that he was posing the most difficult among variants of the same problem with respect to other rings. Nowadays, we know that $\mathrm{H} 10$ as originally posed is unsolvable, but we are in the difficult position of not being able to draw any conclusion about the analog of this problem for, say, the ring $\mathbb{Q}$ of rational numbers.

Martin has been a trailblazer of the field today known as "automated reasoning." The summer of 1954 sees him at work on a JOHNNIAC machine, implementing a logical decision procedure for integer arithmetic. In the late 1950s, a seminal report on computational methods in the propositional calculus arises from his collaboration with Hilary Putnam, the brilliant philosopher with whom Martin enjoyed 


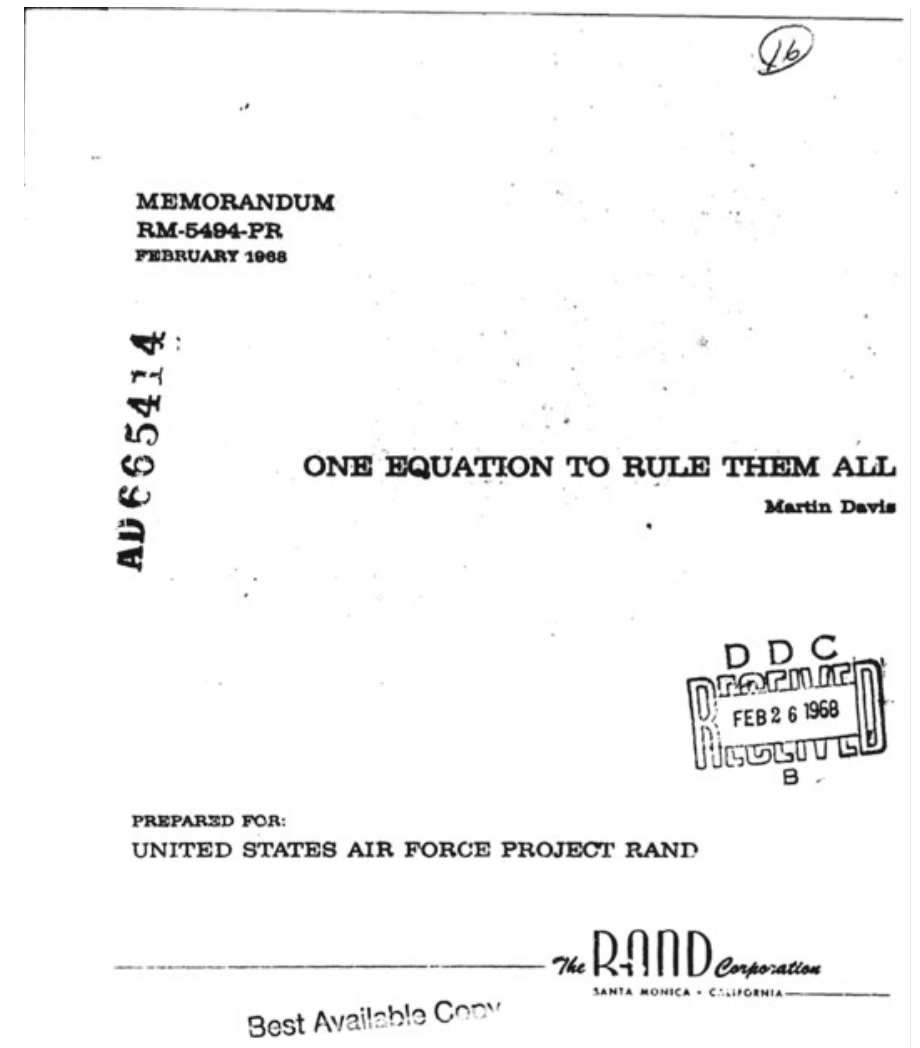

Fig. 4 A report on an intriguing equation

discussing "all day long about everything under the sun, including Hilbert's tenth problem." The Davis-Putnam-Logemann-Loveland procedure, to date so basic in the architecture of fast Boolean satisfiability solvers, was rooted in that study, and Donald Loveland, who contributed to its pioneering implementation in the early 1960s, coauthors in this book, with Ashish Sabharwal and with Professor Bart Selman, a paper reviewing historical developments and the state of the art of propositional theorem provers.

It is slightly less known that in the early 1960s, the most-general unification mechanism for first-order logic was available in the working implementation of Martin's linked conjunct proof procedure, a forerunner of decadelong efforts to automatize reasoning in quantification theory. Unification has evolved, subsequently, into a well-established theory that proceeds hand in hand with the topic of rewriting systems. This is why dedicating a paper on a new trend in this field to 
Martin seemed appropriate: Jörg Siekmann coauthors in this book, with Peter Szabó and Michael Hoche, a survey on "essential unification."

Udi Boker and Nachum Dershowitz, who dedicate an essay on "honest computability" to Martin, contend that "a nefarious representation can turn ... the intractable into trivial," whereas "demanding of an implementation that it also generates its internal representations of the input from an abstract term description of that input ... obviates cheating on complexity problems by giving away the answer in the representation." This attitude is akin to considerations made in the above-cited report by Davis and Putnam (1958) concerning propositional satisfiability, e.g.: "Even if the system has hundreds or thousands of formulas, it can be put into conjunctive normal form 'piece by piece', without any 'multiplying out.' This is a feasible (if laborious) task even for hand computation ..."

The centennial of Frege's Begriffsschrift, Martin reports, "fundamentally changed the direction of my work": Being invited to place some contemporary trends in a proper historical context, he finds "trying to trace the path from ideas and concepts developed by logicians $\cdots$ to their embodiment in software and hardware $\cdots$ endlessly fascinating."

Martin actually cultivated, since long, a keen interest in the history and philosophy of computing: The first edition of The Undecidable, his anthology of basic papers on unsolvable problems and computable functions, is dated 1965. One of Martin's heroes is Alan Mathison Turing; he also devoutly edited the collected works of Emil Leon Post, who had supervised his beginnings in logic at City College. In a recent paper, Martin and Wilfried Sieg have discussed a conceptual confluence between Post and Turing in 1936; in this book, Sieg coauthors with Máté Szabó and Dawn McLaughin, a paper addressing the question: Did Post have Turing's Thesis?

Yiannis N. Moschovakis unravels the history of another crucial confluence of ideas. Stephen C. Kleene, Emil Leon Post, and Andrzej Mostowski had raised questions which would influence profoundly the development of the theory of unsolvability when Martin, in the central part of his Ph.D. thesis, moved "on into the transfinite!", thus playing a very important role in defining natural extensions of the arithmetical hierarchy. The author skillfully alternates notes about the historical development of the subject with some carefully chosen technical details. This makes for a paper which is really a pleasure to read.

Martin once called "attention to the relevance for the foundational problems in quantum theory of some recent mathematical discoveries" arisen from logic. One of the diverse contributions dedicated to Martin, by Andreas Blass and Yuri Gurevich, aims at explaining certain sorts of anyons, "rather mysterious physical phenomena" which may provide a basis for quantum computing, by means of category theory.

Don Perlis's contribution speculates on the concept of infinity and distinguishes several modes of use of infinities in physics. In particular, quantum mechanics, he observes, provides intriguing examples on the subject. Nonstandard analysis - on which Martin wrote a classic — appears to shed light on some such phenomena. 
"Banishing ultrafilters from our consciousness," the title of the paper contributed by Domenico Cantone with the editors of this book, echoes a comment by Martin in his Applied Nonstandard Analysis (1977). Martin then pointed out that the intricacies of the ultrapower construction of a nonstandard universe can be completely forgotten in favor of a few principles relating standard/nonstandard, internal/external objects. Bearing Martin's motto in mind, this paper recounts, and aided by a proof-checker embodying constructs for proof engineering, the authors have undertaken a verification of key results of the nonstandard approach to analysis.

The reader will also attend, inside this volume, Martin Davis and Hilary Putnam resuming some threads of their juvenile philosophical discussion. Martin has recently written about realism in mathematics (partly because Harvey Friedman had judged him "an extreme Platonist"), and Hilary cannot resist to amicably respond to his fascinating essay Pragmatic Platonism (also included in this book) and to discuss the relation between Martin's view and the views Hilary defends. In his turn, Martin comments on Hilary's remarks on his essay and takes the opportunity to say a little more about his view about certain topics such as mathematics and natural science, and new axioms for set theory.

The first chapter is an autobiographic essay by the eminent logician to whom the entire book is devoted. An earlier version of this essay, published in 1999, was titled "From Logic to Computer Science and Back." Martin reports that his debut as a computer programmer takes place in 1951, "without [him] realizing it," while he - a recent Ph.D. from Princeton, teaching recursive function theory at ChampaignUrbana-is designing Turing machines; he then gets recruited for a project on an automated system for navigating airplanes, with the task of writing code for an ORDVAC machine. Short afterward, Martin conceives the idea of writing his first book on computability; then, planning an extended visit to the Institute for Advanced Study in Princeton, he proposes to work on connections between logic and information theory. In the following decades, he frequently moves across the USA, teaching in various academic institutions and working on computability, on Hilbert's tenth problem, on computational logic, etc.

Multifaceted life and publications, but a substantial unity: in the new title chosen for his enriched autobiography, Martin regards himself simply as a logician.

Trieste and Udine

February 2016
Eugenio G. Omodeo

Alberto Policriti 


\section{Acknowledgements}

The editors gratefully acknowledge invaluable help from Martin Davis and Yuri Matiyasevich for many aspects of the preparation of this book. Martin, in particular, supplied old manuscripts (his Ph.D. thesis, the research reports jointly written with Hilary Putnam which are included as appendices) and re-read certain parts of the draft of this book. Yuri offered great support for the preparation of Martin Davis's bibliography (Chap. 16).

Dominique Pastre (emeritus professor of computer science at UFR de mathématiques et informatique, Université Paris Descartes) kindly accepted to be an "anonymity server" to referees of her choice.

The anonymous second readers for this volume include the following:

Samson Abramsky, Department of Computer Science University of Oxford; Johan van Benthem, University of Amsterdam and Stanford University;

Alessandro Berarducci, Dipartimento di Matematica, Università di Pisa;

Maria Paola Bonacina, Dip. di Informatica, Università degli Studi di Verona;

Patrick Cégielski, Université Paris Est Créteil;

Pietro Corvaja, Dip. di Matematica e Informatica, Università di Udine;

Liesbeth De Mol, Université de Lille 3, Villeneuve d'Ascq Cedex;

Francesco M. Donini, Università della Tuscia, Viterbo;

David Finkelstein, School of Physics, Georgia Institute of Technology, Atlanta; Andrea Formisano, Università di Perugia;

Miriam Franchella, Dipartimento di Filosofia, Università degli Studi di Milano; Jean-Pierre Keller, Sarl Kepler, Paris;

Gabriele Lolli, Scuola Normale Superiore di Pisa;

Stefano Mancini, School of Science and Technology, University of Camerino; Alberto Marcone, Dip. di Matematica e Informatica, Università di Udine;

Alberto Martelli, Dipartimento di Informatica, Università degli Studi di Torino; Daniele Mundici, Dept. of Mathematics and Computer Science "Ulisse Dini," University of Florence; 
Andrea Sorbi, Dip. di Ingegneria dell'Informazione e Scienze Matematiche, Università degli Studi di Siena; and

Carlo Toffalori, Dip. Matematica e Fisica, Univ. of Camerino. 


\section{Contents}

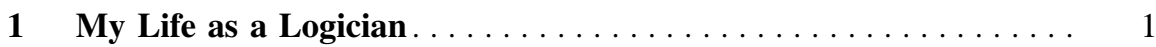
Martin Davis

2 Martin Davis and Hilbert's Tenth Problem. . . . . . . . . . . 35 Yuri Matiyasevich

3 Extensions of Hilbert's Tenth Problem: Definability and

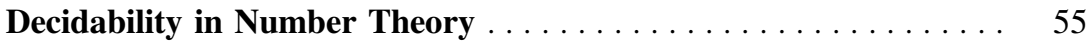
Alexandra Shlapentokh

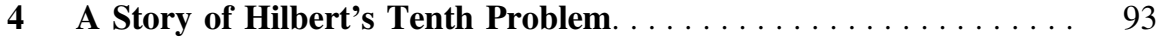
Laura Elena Morales Guerrero

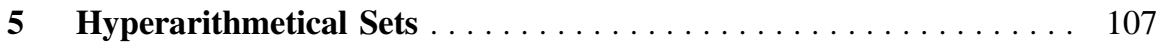
Yiannis N. Moschovakis

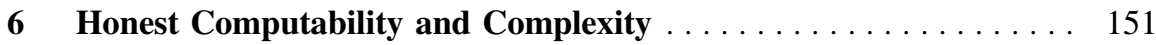
Udi Boker and Nachum Dershowitz

7 Why Post Did [Not] Have Turing's Thesis . . . . . . . . . . . . . 175 Wilfried Sieg, Máté Szabó and Dawn McLaughlin

8 On Quantum Computation, Anyons, and Categories . . . . . . . . . 209 Andreas Blass and Yuri Gurevich

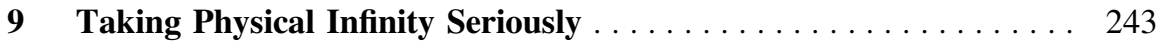
Don Perlis

10 Banishing Ultrafilters from Our Consciousness. . . . . . . . . . . . 255 Domenico Cantone, Eugenio G. Omodeo and Alberto Policriti

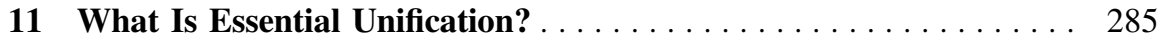
Peter Szabo, Jörg Siekmann and Michael Hoche 
12 DPLL: The Core of Modern Satisfiability Solvers . . . . . . . . . . 315 Donald Loveland, Ashish Sabharwal and Bart Selman

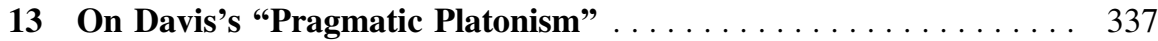
Hilary Putnam

14 Pragmatic Platonism . . . . . . . . . . . . . . . . . . . . . . . 349 Martin Davis

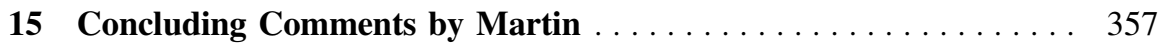
Martin Davis

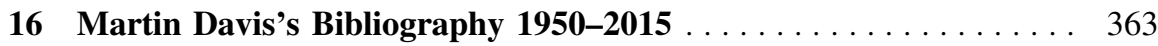
Eugenio G. Omodeo

Appendix A: "Feasible Computational Methods in the Propositional Calculus", the Seminal Report by M. Davis and H. Putnam . . . . . . . . 371

Appendix B: "Research on Hilbert's Tenth Problem", the Original Paper by M. Davis and H. Putnam . . . . . . . . . . . . . . . . . . . . 409

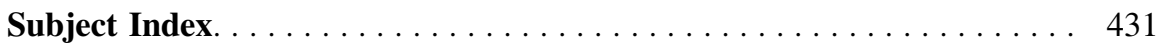

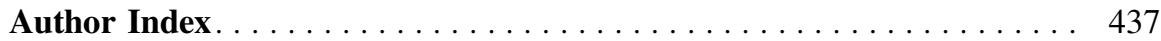




\section{Contributors and Editors}

\section{Contributors}

Andreas Blass is a professor of mathematics at the University of Michigan. He received his Ph.D. degree from Harvard University in 1970 and has been at the University of Michigan ever since except for brief leaves. He has also been a visiting researcher at Microsoft Research during one of those leaves and during each of the last 13 summers. His primary research area is set theory, but he has also worked in other branches of logic, in theoretical computer science, and in finite combinatorics. He is a fellow of the American Mathematical Society.

Udi Boker is a senior researcher in the Interdisciplinary Center (IDC), Herzliya, working in the field of logic and verification. His main research areas concern the foundations of computation, temporal logic, and automata.

Domenico Cantone is a professor of computer science since 1990. He is currently at the University of Catania, Italy, where he moved from the University of L'Aquila, Italy, in 1991. He received his Ph.D. degree from New York University in 1987, under the supervision of Prof. Jacob T. Schwartz. Since 1995, he has been a member of the Board of Directors of the journal "Le Matematiche." His main scientific interests include the following: computable set theory, automated deduction in various mathematical theories, description logic, string matching and algorithmic engineering, and, more recently, rational choice theory from a logical point of view. In the field of computable set theory, he has coauthored three monographs: Computable Set Theory (Clarendon Press, 1989), Set Theory for Computing-From Decision Procedures to Declarative Programming with Sets (Springer, 2001), and Computational Logic and Set Theory: Applying Formalized Logic to Analysis, (Springer, 2011).

Nachum Dershowitz is professor of computational logic at Tel Aviv University, where he has been since 1998. Prior to that, he was on the faculty of the University of Illinois at Urbana-Champaign. He coauthored the book, Calendrical Calculations (Cambridge University Press, 1997), with Edward Reingold, which won Choice's 
Outstanding Academic Title Award (2002) and is going into its fourth edition. He is also the author of The Evolution of Programs (Birkhäuser, 1983), coauthor of Calendrical Tabulations (Cambridge University Press, 2002), and editor of a dozen other volumes. His research interests include foundations of computing, computational logic, computational humanities, and combinatorial enumeration. He has received the Herbrand Award for Distinguished Contributions to Automated Reasoning (2011), the Logic in Computer Science (LICS) Test-of-Time Award (2006), the Rewriting Techniques and Applications (RTA) Test-of-Time Award (2014), and the Conference on Automated Deduction (CADE) Skolem Award (2015) and was elected to Academia Europaea in 2013.

Yuri Gurevich is a principal researcher at Microsoft Research in Redmond, Washington, USA. He is also Professor Emeritus at the University of Michigan, ACM Fellow, Guggenheim Fellow, EATCS Fellow, a foreign member of Academia Europaea, and Dr. Honoris Causa of a Belgian and Russian universities.

Michael Hoche studied computer science at University of Stuttgart with visits at József Attila University, Szeged, and University Pierre and Marie Curie, Paris. He is currently working at Airbus Defense and Space, where he serves as analyst and technologist in advanced computer science and its applications. He holds a Ph.D. in computer science, which he obtained from University of Stuttgart. His current interest comprises intelligent systems applications with a focus on data integration, machine learning, and networks. Most recently, he contributes to the creation of the research agenda of Airbus Defense and Space.

Donald Loveland attended Oberlin College, MIT, and New York University (NYU) where he wrote his Ph.D. thesis under Martin Davis. He was on the faculties of NYU (Bronx Campus) where Martin was briefly a colleague, CMU, and Duke University where he was the first chairman of the Computer Science Department. The author of Automated Theorem Proving: a Logical Basis (1978) is also one of three authors of the undergraduate textbook Three Views of Logic (2014). He is author or coauthor of papers in areas that include automated deduction (primary area), complexity theory, testing procedures, logic programming, and expert systems; an ACM Fellow; and an AAAI Fellow, and he also received the Herbrand Award (2001). He is now retired.

Yuri Matiyasevich has got (Russian analog of) Ph.D. from Leningrad (now St. Petersburg) Division of Steklov Mathematical Institute in 1970 and ever since works there, today as the Head of Laboratory of mathematical logic. He is mostly known for his contribution to the (negative) solution of Hilbert's tenth problem, about which he wrote a book translated into English and French. He worked also in theoretical computer science, graph theory, and number theory. He is full member of Russian Academy of Sciences, corresponding member of Bavarian Academy of Sciences, and member of Academia Europaea and Docteur Honoris Causa de l'Université d'Auvergne et de Université Pierre et Marie Curie (Paris-6), France. 
Dawn McLaughlin is a Ph.D. candidate in the philosophy department at Carnegie Mellon University and did early work on Emil Post; however, her academic focus is now on logic education. She is a collaborator on Wilfried Sieg's AProS project, in particular regarding the online logic course Logic and Proofs; she is the lead developer of the LogicLab. Her dissertation is exploring the history and practice of technology supported learning of logic.

Laura Elena Morales Guerrero was the second in a Mexican family of seven children living in Tampico, Mexico. After receiving her undergraduate degree at the University of Wisconsin, she began graduate studies in physics and mathematics at the Centro de Investigación y Estudios Avanzados (Cinvestav, IPN) in México City. Her doctorate under Jerzy Plebański involved finding exact solutions of the field equations of Einstein's general relativity. She worked in the nuclear engineering industry in Mexico and The Netherlands. While rearing her twin daughters, she started a successful retail business in Mexico City. She remains an independent researcher, and her most recent interests are in the history of mathematics, especially logic and number theory. She connected with Martin Davis through the email list HM (History of Mathematics) and arranged for him to lecture at the Universidad Nacional Autónoma de México (UNAM) where she was a researcher at the time. His talk stimulated her interest in Hilbert's tenth problem and led her to write the essay on the subject in this book. In his preface to her still unpublished book on the ancient problem of squaring the circle, he wrote, "In this charming book, Dra. Morales has traced the history of this problem, pausing along the way to explore many fascinating sidelights."

Yiannis N. Moschovakis emigrated to the USA from Greece in 1956. After four years at MIT, three years at the University of Wisconsin and one year at Harvard, he moved to UCLA in 1964 and has been there ever since, with long, annual sojourns to Greece including a halftime position at the University of Athens (UOA) from 1996 until 2005. He has written two monographs and a textbook and has supervised or cosupervised the doctoral dissertations of 22 students from the mathematics and computer science Departments of UCLA, UOA, and the Graduate Program for logic, algorithms, and computation in Athens. He retired in 2010 but has continued teaching part-time as a Distinguished Research Professor.

Moschovakis has worked in abstract and higher-type recursion; in classical and effective descriptive set theory, including the consequences of determinacy hypotheses; and in philosophical logic, especially the logic of meaning and the foundations of the theory of algorithms.

Don Perlis is a professor of computer science at the University of Maryland, College Park. He has Ph.D.s in mathematics (NYU, 1972) and computer science (Rochester, 1981). While his interests span a wide variety of areas, he recently has come to realize that most of his work relates to self-reference in one form or another, whether in logic, artificial intelligence, cognitive science, or philosophy. 
Hilary Putnam is Cogan University Professor Emeritus at Harvard University. His most recent publications include philosophy in an age of science (ed. by M. De Caro and D. Macarthur, Harvard UP 2012) and naturalism, realism, and normativity (ed. by M. De Caro, Harvard UP 2016). He is the subject of The Philosophy of Hilary Putnam (ed. by R.E. Auxier, D.R. Anderson, and L.E. Hahn, Library of Living Philosophers, Open Court 2015). He holds 12 honorary degrees and is a past president of the American Philosophical Association, a fellow of the American Academy of Arts and Sciences, and a corresponding fellow of the British Academy and of the French Académie des Sciences Politiques et Morales. Recently, he has been awarded the Prometheus Prize, the Rolf Schock Prize in logic and philosophy, the Lauener Prize for analytical philosophy, and the Nicholas Rescher Prize for systematic philosophy. His interests cover most philosophical areas.

Ashish Sabharwal investigates scalable and robust methods for probabilistic and combinatorial inference, graphical models, and discrete optimization, as a research scientist at the Allen Institute for AI (AI2), especially as they apply to assessing machine intelligence through standardized examinations in science and math. Prior to joining AI2, Ashish spent over three years at IBM Watson and five years at Cornell University, after obtaining his Ph.D. from the University of Washington in 2005. Ashish has coauthored over 70 publications, been part of winning teams in international SAT competitions, and received five best paper awards and runner-up prizes at venues such as AAAI, IJCAI, and UAI.

Bart Selman is a professor of computer science at Cornell University. He was previously at AT\&T Bell Laboratories. He specializes in artificial intelligence, with an emphasis on efficient reasoning procedures, planning, knowledge representation, and connections between computer science and statistical physics. He has (co) authored over 150 publications, including six best paper awards. His papers have appeared in venues spanning Nature, Science, Proc. Natl. Acad. of Sci., and a variety of conferences and journals in $\mathrm{AI}$ and computer science. He has received the Cornell Stephen Miles Excellence in Teaching Award, the Cornell Outstanding Educator Award, an NSF Career Award, and an Alfred P. Sloan Research Fellowship. He is a fellow of the American Association for Artificial Intelligence (AAAI) and a fellow of the American Association for the Advancement of Science (AAAS). He received the inaugural IJCAI John McCarthy award in 2015.

Alexandra Shlapentokh is a professor of mathematics in East Carolina University in Greenville, NC. She got her Ph.D. in mathematics in NYU in 1988 under Professor Harold N. Shapiro. In graduate school, Alexandra Shlapentokh also had the privilege of having Martin Davis as one of her professors. It is in his class that she was introduced for the first time to Hilbert's tenth problem which became one of her lifelong interests, eventually encompassing many questions of definability and computability in number theory. She described some of the developments in this thriving field in her book: "Hilbert's Tenth Problem: Diophantine Classes and Other Extensions to Global Fields" (Cambridge University Press). 
Wilfried Sieg is a patrick suppes professor of Philosophy at Carnegie Mellon University and a fellow of the American Academy of Arts and Sciences. He joined Carnegie Mellon's faculty in 1985 as a founding member of the University's Philosophy Department and served as its head from 1994 to 2005 . He is internationally known for mathematical work in proof and computation theory, historical work on modern logic and mathematics, and philosophical essays on the nature of mathematics. A collection of essays joining the three aspects of his research was published as Hilbert's Programs and Beyond (Oxford University Press, 2013).

Jörg Siekmann studied mathematics at Göttingen University and computer science at Essex University, England, where he received his Ph.D. in unification theory in 1976. He was a scientific assistant at Karlsruhe University and became professor for Artificial Intelligence at Kaiserslautern in 1983. In 1991, he was appointed as a full professor for computer science and AI at Saarbrücken University. He founded with friends the German Research Centre for Artificial Intelligence (DFKI) and became one of its directors. He is now a senior professor at the computer science department and DFKI in Saarbrücken.

Máté Szabó is a Ph.D. student in the philosophy department at Carnegie Mellon University. His main interests concern the history and philosophy of computing, in particular the interpretation of Gödel's Incompleteness Theorems, Church's and Turing's Undecidability Theorems, and of the Church-Turing Thesis. Related to these issues, he has been exploring the life and work of Emil Post and László Kalmár. He is writing his dissertation on human and machine computation with Wilfried Sieg as his advisor.

Peter Szabó studied computer science at the University of Karlsruhe where he received his diploma in 1975 and worked there afterward as a scientific assistant. He received his Ph.D. in unification theory from Karlsruhe University in 1982. From 1983 to 2011, he was a research engineer for software in the R\&D department of the German Telecommunication company SEL, which later became part of Alcatel-Lucent. Since 2003, he is a voluntary member of our research team dealing with unification theory.

Andreas Blass Mathematics Department, University of Michigan, Ann Arbor, MI, USA

Udi Boker School of Computer Science, Interdisciplinary Center, Herzliya, Israel Domenico Cantone DMI, Università di Catania, Catania, Italy

Martin Davis Department of Mathematics, University of California, Berkeley, CA, USA; Courant Institute of Mathematical Sciences, New York University, New York, NY, USA

Nachum Dershowitz School of Computer Science, Tel Aviv University, Ramat Aviv, Israel

Yuri Gurevich Microsoft Research, Redmond, WA, USA 
Michael Hoche Airbus Defense and Space, Immenstaad, Germany

Donald Loveland Duke University, Durham, USA

Yuri Matiyasevich Laboratory of Mathematical Logic, St. Petersburg Department of V.A. Steklov Institute of Mathematics (POMI), Russian Academy of Sciences, St. petersburg, Russia

Dawn McLaughlin Carnegie Mellon University, Pittsburgh, USA

Laura Elena Morales Guerrero Centro de Investigación y de Estudios Avanzados del Instituto Politécnico Nacional in Zacatenco, Ciudad de México, Mexico

Yiannis N. Moschovakis Department of Mathematics, University of California, Los Angeles, USA

Eugenio G. Omodeo DMG/DMI, Università di Trieste, Trieste, Italy

Don Perlis University of Maryland, College Park, USA

Alberto Policriti DMIF, Università di Udine, Udine, Italy

Hilary Putnam University of Harvard, Cambridge, USA

Ashish Sabharwal Allen Institute for AI, Seattle, USA

Bart Selman Cornell University, Ithaca, USA

Alexandra Shlapentokh East Carolina University, Greenville, USA

Wilfried Sieg Carnegie Mellon University, Pittsburgh, USA

Jörg Siekmann Saarland University/DFKI, Saarbrücken, Germany

Peter Szabo Pforzheim, Germany

Máté Szabó Carnegie Mellon University, Pittsburgh, USA

\section{About the Editors}

Eugenio G. Omodeo studied mathematics at the University of Padua and then computer science at the New York University, GSAS, where he earned Ph.D. (1984) under the supervision of Martin Davis. From 1981 to 1989, he was employed by companies belonging to ENI, the National Hydrocarbon Group of Italy: There, after 1984, he coordinated R\&D activities of Enidata in various projects funded by the European Commission (CEC), mainly focused on declarative programming and on quick prototyping. From 1989 to present, he has been a professor in various Italian universities (Udine, "La Sapienza" of Rome, Salerno, L'Aquila, Trieste). He has contributed to computational logic with the discovery of inference methods based on set theory, some of which have been implemented in a 
large-scale proof verifier developed with Jacob T. Schwartz (NYU). He coauthored three scientific monographs on computable set theory.

Alberto Policriti received his degree in mathematics from the Universtiy of Turin in 1984 and his Ph.D. in computer science under the supervision of M. Davis in 1990. From 1989, he is at the University of Udine, where he is currently professor of computer science at the Department of Mathematics, Computer Science, and Physics. His main research interests are related to computational logic and algorithms: set-theoretic and combinatorial algorithms and problems, modal and temporal logics, and algorithms and models for bioinformatics. He has coauthored two monographs and has supervised or co-supervised 15 doctoral dissertations in Logic, Algorithms, and Bioinformatics. He is one of the four founders of the "Istituto di Genomica Applicata," has been member of the scientific committee of GNCS - "Istituto di Alta Matematica," and he is currently member of the scientific committee of the EATCS. 